\title{
Neutrophil-to-Lymphocyte Ratio After Definitive Concurrent Chemoradiotherapy Predicts Survival in Patients With Esophageal Squamous Cell Carcinoma
}

\author{
HYEON KANG KOH ${ }^{1 *}$, YOUNGHEE PARK ${ }^{2 *}$, TAERYOOL $\mathrm{KOO}^{3}$, HAE JIN PARK ${ }^{4}$, \\ ME YEON LEE ${ }^{3}$, AH RAM CHANG ${ }^{2}$, SEMIE HONG ${ }^{1}$ and HOONSIK BAE ${ }^{3}$ \\ ${ }^{1}$ Department of Radiation Oncology, Konkuk University School of Medicine \\ and Konkuk University Medical Center, Seoul, Republic of Korea; \\ ${ }^{2}$ Department of Radiation Oncology/CyberKnife Center, \\ Soonchunhyang University Seoul Hospital, Seoul, Republic of Korea; \\ ${ }^{3}$ Department of Radiation Oncology, Hallym University Sacred Heart Hospital, \\ Hallym University College of Medicine, Anyang, Republic of Korea; \\ ${ }^{4}$ Department of Radiation Oncology, Hanyang University College of Medicine, Seoul, Republic of Korea
}

\begin{abstract}
Background/Aim: Lymphocyte-to-monocyte ratio, neutrophil-to-lymphocyte ratio, and platelet-to-lymphocyte ratio represent systemic immune-inflammatory responses. We evaluated the association between immune-inflammatory cell ratios and prognosis in esophageal squamous cell carcinoma (ESCC) patients who underwent definitive concurrent chemoradiotherapy (dCCRT). Patients and Methods: Medical records of 68 ESCC patients in three institutions who underwent dCCRT between 2006 and 2017 were reviewed. The immune-inflammatory cell ratios were calculated before and after dCCRT. Results: The median follow-up time was 11.4 months. The 3-year overall survival (OS) rate was 21.6\%. Among the immune-inflammatory cell ratios, lower post-dCCRT neutrophil-to-lymphocyte ratio (NLRpost) was associated with better OS (median $15.2 \mathrm{vs.}$ 9.7 months, $p=0.030)$. Patients with lower NLRpost had more improved OS when adjuvant chemotherapy was administered following dCCRT (median $16.6 \mathrm{vs} .4 .8$ months,
\end{abstract}

This article is freely accessible online.

*These Authors contributed equally to this study.

Correspondence to: Taeryool Koo, MD, Ph.D., Department of Radiation Oncology, Hallym University Sacred Heart Hospital, Hallym University College of Medicine, 22, Gwanpyeong-ro 170beon-gil, Dongan-gu, Anyang-si, Gyeonggi-do, 14068, Republic of Korea. Tel: +82 313803901, Fax: +82 313803913, e-mail: kootaeryool@hallym.or.kr

Key Words: Chemoradiotherapy, esophageal squamous cell carcinoma, neutrophil-to-lymphocyte ratio, inflammation. $p<0.001)$. Conclusion: NLRpost may be useful in predicting OS in ESCC patients after dCCRT. Furthermore, NLRpost might play a role in establishing adjuvant therapy plans following dCCRT.

Immune cells play important roles in the tumor microenvironment (TME). In the TME, tumor cells can have conflicting courses depending on the immune cells that interact with each other. Innate immune cells execute antitumor activity by initiating inflammation after sensing pathogen-associated or danger-associated molecular patterns. However, malignant cells are not eradicated in the TME under chronic inflammation (1). Chronic inflammation contributes to the proliferation and survival of malignant cells, angiogenesis, and metastasis (2). The inhibited antitumor immunity was caused by immunosuppressive cells, mitogenic growth factors, and cytokines, such as myeloidderived suppressor cells, regulatory T cells, and transforming growth factor- $\beta$ (3).

Evaluation of TME could be helpful for the assessment of prognosis in cancer patients. However, there would be difficulty in acquiring tissues from the tumor origin given the patient's convenience and anatomical risks. Lymphocytes, monocytes, neutrophils, and platelets are essential components of inflammation and immune responses, which can be tested easily and economically using peripheral blood. Lymphocyte-based indices, such as lymphocyte-to-monocyte ratio (LMR), neutrophil-to-lymphocyte ratio (NLR), and platelet-to-lymphocyte ratio (PLR), are known to be surrogate markers of systemic immune-inflammatory responses. In many solid tumors, the prognostic value of LMR, NLR, and PLR has been reported (4-7). 
Table I. Patient characteristics and univariate analysis.

\begin{tabular}{|c|c|c|c|c|c|}
\hline Variables & & $\mathrm{N}$ & $(\%)$ & Median OS (months) & $p$-Value \\
\hline \multirow[t]{2}{*}{ Age (year) } & $\leq 65$ & 31 & $(45.6)$ & 17.4 & 0.031 \\
\hline & $>65$ & 37 & $(54.4)$ & 10.0 & \\
\hline \multirow[t]{2}{*}{ Gender } & Female & 4 & $(5.9)$ & 13.7 & 0.707 \\
\hline & Male & 64 & $(94.1)$ & 11.1 & \\
\hline \multirow[t]{2}{*}{ Location } & Lower & 18 & $(26.5)$ & 12.3 & 0.256 \\
\hline & Upper-middle & 50 & $(73.5)$ & 10.9 & \\
\hline \multirow[t]{2}{*}{ T stage } & $\mathrm{T} 1-2$ & 17 & $(25.0)$ & 96.0 & 0.007 \\
\hline & T3-4 & 51 & $(75.0)$ & 10.4 & \\
\hline \multirow[t]{2}{*}{$\mathrm{N}$ stage } & N0-1 & 37 & $(54.4)$ & 14.6 & 0.001 \\
\hline & $\mathrm{N} 2-3$ & 31 & $(45.6)$ & 9.1 & \\
\hline \multirow[t]{2}{*}{ Performance status } & ECOG $0-1$ & 54 & (79.4) & 11.4 & 0.710 \\
\hline & ECOG 2-3 & 14 & $(20.6)$ & 12.7 & \\
\hline \multirow[t]{2}{*}{ BED (cGy) } & $\leq 6372$ & 32 & $(47.1)$ & 11.1 & 0.357 \\
\hline & $>6372$ & 36 & $(52.9)$ & 13.6 & \\
\hline \multirow{2}{*}{ Adjuvant chemotherapy } & No & 14 & $(20.6)$ & 4.5 & $<0.001$ \\
\hline & Yes & 54 & (79.4) & 14.6 & \\
\hline \multirow[t]{2}{*}{ LMRpre } & $\leq 5.75$ & 60 & $(88.2)$ & 10.7 & 0.020 \\
\hline & $>5.75$ & 8 & $(11.8)$ & Not reached* & \\
\hline \multirow[t]{2}{*}{ NLRpre } & $\leq 2.50$ & 34 & $(50.0)$ & 16.6 & 0.012 \\
\hline & $>2.50$ & 34 & $(50.0)$ & 9.7 & \\
\hline \multirow[t]{2}{*}{ PLRpre } & $\leq 83.1$ & 8 & $(11.8)$ & 6.7 & 0.067 \\
\hline & $>83.1$ & 60 & $(88.2)$ & 13.4 & \\
\hline \multirow[t]{2}{*}{ LMRpost } & $\leq 2.51$ & 53 & (77.9) & 10.4 & 0.018 \\
\hline & $>2.51$ & 15 & $(22.1)$ & 33.1 & \\
\hline \multirow[t]{2}{*}{ NLRpost } & $\leq 5.44$ & 42 & (61.8) & 15.2 & 0.030 \\
\hline & $>5.44$ & 26 & $(38.2)$ & 9.7 & \\
\hline \multirow[t]{2}{*}{ PLRpost } & $\leq 372.2$ & 39 & (57.4) & 13.3 & 0.022 \\
\hline & $>372.2$ & 29 & (42.6) & 10.0 & \\
\hline
\end{tabular}

*The 3-year overall survival rate is $56.2 \%$. OS: Overall survival; ECOG: Eastern Cooperative Oncology Group; BED: biologically effective dose; LMR: lymphocyte-to-monocyte ratio; NLR: neutrophil-to-lymphocyte ratio; PLR: platelet-to-lymphocyte ratio

Definitive concurrent chemoradiotherapy (dCCRT) is the mainstay treatment for esophageal squamous cell carcinoma (ESCC). Radiotherapy (RT) reduces peripheral blood cell counts, especially lymphocytes, which are more radiosensitive than other leukocytes (8). Chemotherapy adversely affects the hematopoietic system, and neutropenia is one of the most serious hematologic toxicities of the treatment (9). With regards to the effect of RT and chemotherapy on immune-inflammatory responses, we aimed to compare the predictive value of LMR, NLR, and PLR measured before and after dCCRT in ESCC patients.

\section{Patients and Methods}

This study was performed by three institutions under the approval of the Institutional Review Board (IRB) of each participating hospital. Each IRB approved a waiver for informed consent. Details regarding data collection are described in a previous report (10). Information on 73 patients who underwent dCCRT for ESCC between 2006 and 2017 were reviewed. We collected lymphocyte, monocyte, neutrophil, and platelet counts within a week before the start and after the completion of RT. One patient with insufficient laboratory data was excluded. Given the potential effects of chemotherapy on the blood cell count, four patients who received chemotherapy prior to the start of RT were excluded. Finally, 68 patients were included in the present study.

The data we collected for this study comprised patient and tumor characteristics, clinical information, treatment details, and the blood cell count. The locations of the primary tumors were categorized according to their distance from the upper incisors: upper thoracic $(\leq 24 \mathrm{~cm}$ from the upper incisor), middle thoracic $(\leq 32 \mathrm{~cm}$ from the upper incisor), and lower thoracic ( $>32 \mathrm{~cm}$ from the upper incisor). The leukocyte count was measured in cells per microliter (cells $/ \mu \mathrm{l})$. LMR was defined as the count of lymphocytes over monocytes, NLR as neutrophils over lymphocytes, and PLR as platelets over lymphocytes.

We defined overall survival (OS) as the time from the pathologic diagnosis of ESCC to the last follow-up or death. Survival rates were estimated using the Kaplan-Meier method. The log-rank test and Cox proportional-hazards model were used for univariate and multivariate analyses, respectively. Differences in categorical variables were verified using Pearson's chi-square test or Fisher's exact test. Continuous variables, including RT dose, LMR, NLR, and PLR, were divided into two groups using the maximally selected rank method. We selected cutoff values that best separated OS. A $p$-value of $<0.05$ was considered statistically significant. We used R 3.6.2 (R Development Core Team, Vienna, Austria) for the statistical analysis (11). 

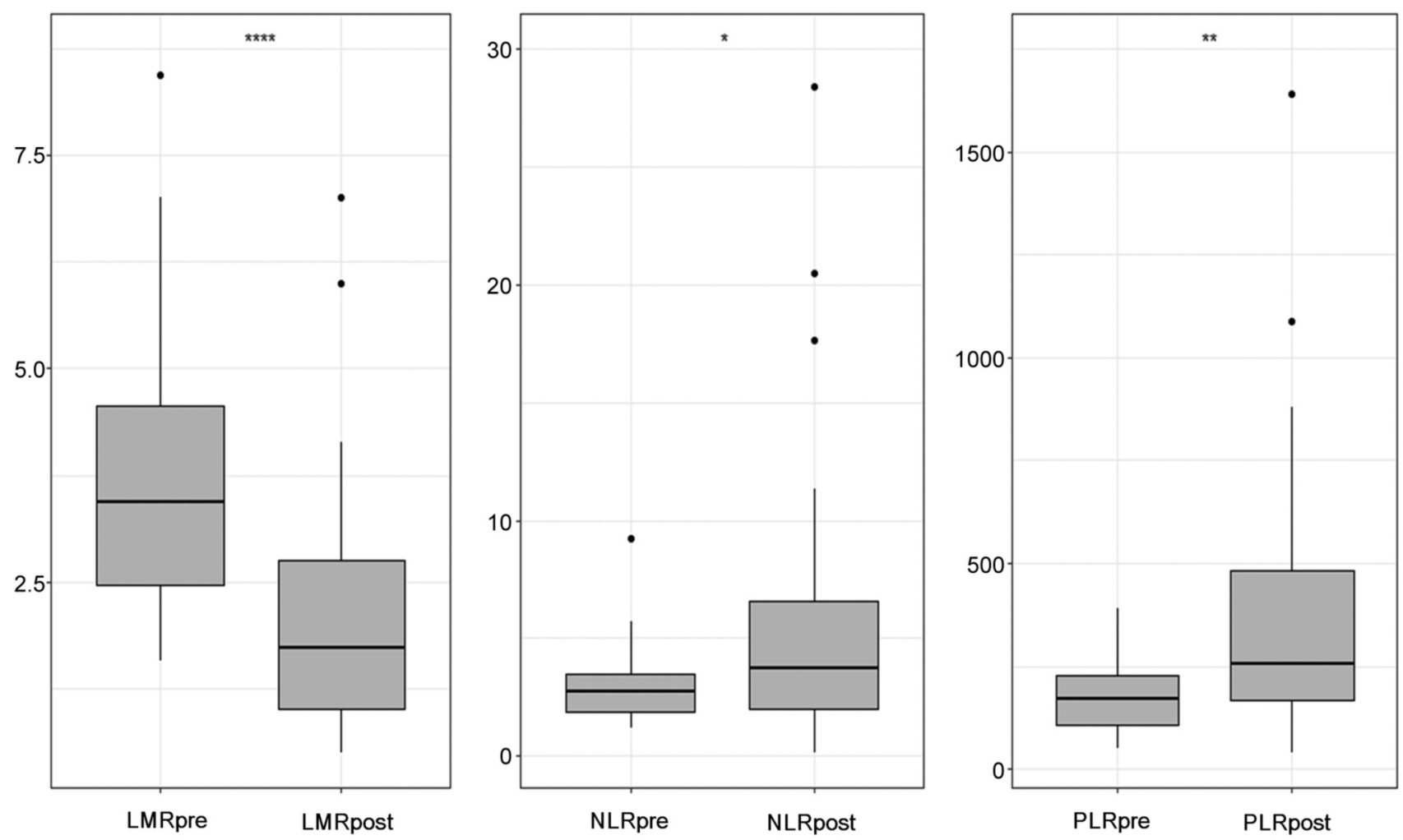

Figure 1. Blox plots for lymphocyte-to-monocyte ratio (LMR), neutrophil-to-lymphocyte ratio (NLR), and platelet-to-lymphocyte ratio (PLR). The middle horizontal lines indicate the median values, and the upper and lower lines of the box indicate the 75th and 25th percentiles, respectively $\left({ }^{*} p \leq 0.05, * * p \leq 0.01, * * * p \leq 0.001\right.$, and $\left.* * * * p \leq 0.0001\right)$.

We categorized adverse events according to the Common Terminology Criteria for Adverse Events (CTCAE) version 5. Acute and chronic adverse effects were defined as adverse effects within 3 months and 4 months after the completion of dCCRT, respectively.

\section{Results}

Patients and treatments. The median age was 66 years (range $=47-84$ years). The location of the primary tumor was upper thoracic in $16(24 \%)$, middle thoracic in $34(50 \%)$, and lower thoracic in $18(26 \%)$ patients. RT was performed using three-dimensional conformal RT (58 patients, $85 \%$ ) or intensity-modulated RT (10 patients, $15 \%$ ). The median total dose was 55.8 Gy (range=50-70 Gy) and the median daily dose was $1.8 \mathrm{~Gy}$ (range=1.8-2 Gy). The median biologically effective dose (BED) was 65.8 Gy (range=59.5-84 Gy) with $\alpha / \beta=10$. Concurrent with RT, a combination of 5-fluorouracil (5-FU) and cisplatin was administered in 63 patients $(93 \%)$. On the other hand, 5-FU alone was administered in 4 patients and cisplatin alone was administered in 1 patient. After the completion of RT, 54 patients (79\%) underwent adjuvant chemotherapy with a combination of 5-FU and cisplatin. Details of patient characteristics and treatment are listed in Table I.
LMR, NLR, and PLR. Before RT, the median absolute count of lymphocytes was 1,538 (range $=400-3,178$ ), monocytes 444 (range $=41-1,087)$, neutrophils 3,710 (range $=1,536-$ 10,552 ), and platelets 254,500 (range $=100,000-476,000$ ). After RT, the median absolute count of lymphocytes was 600 $($ range $=30-2,600)$, monocytes $400 \quad($ range $=11-1,101)$, neutrophils 2,936 (range $=54-10,040)$, and platelets 196,000 (range $=43,000-657,000)$.

LMR, NLR, and PLR showed significant changes through RT (Figure 1). Before RT, the median ratio was LMR 3.64 (range=1.14-13.80), NLR 2.58 (range=1.13-13.30), and PLR 163.2 (range $=50.8-647.5$ ). After RT, the median ratio was LMR 1.35 (range=0.33-7.00), NLR 4.77 (range=0.15-52.26), and PLR 330.7 (range=39.6-6666.7). The cut-off values calculated before the start of RT were 5.75 for LMR (LMRpre), 2.50 for NLR (NLRpre), and 83.1 for PLR (PLRpre). The cutoff values calculated after the completion of RT were 2.51 for LMR (LMRpost), 5.44 for NLR (NLRpost), and 372.2 for PLR (PLRpost).

Survival outcomes and prognostic/predictive factors. The median follow-up time was 11.4 months (range=2.1-108.8 months) and the median OS was 11.4 months. The OS rates 


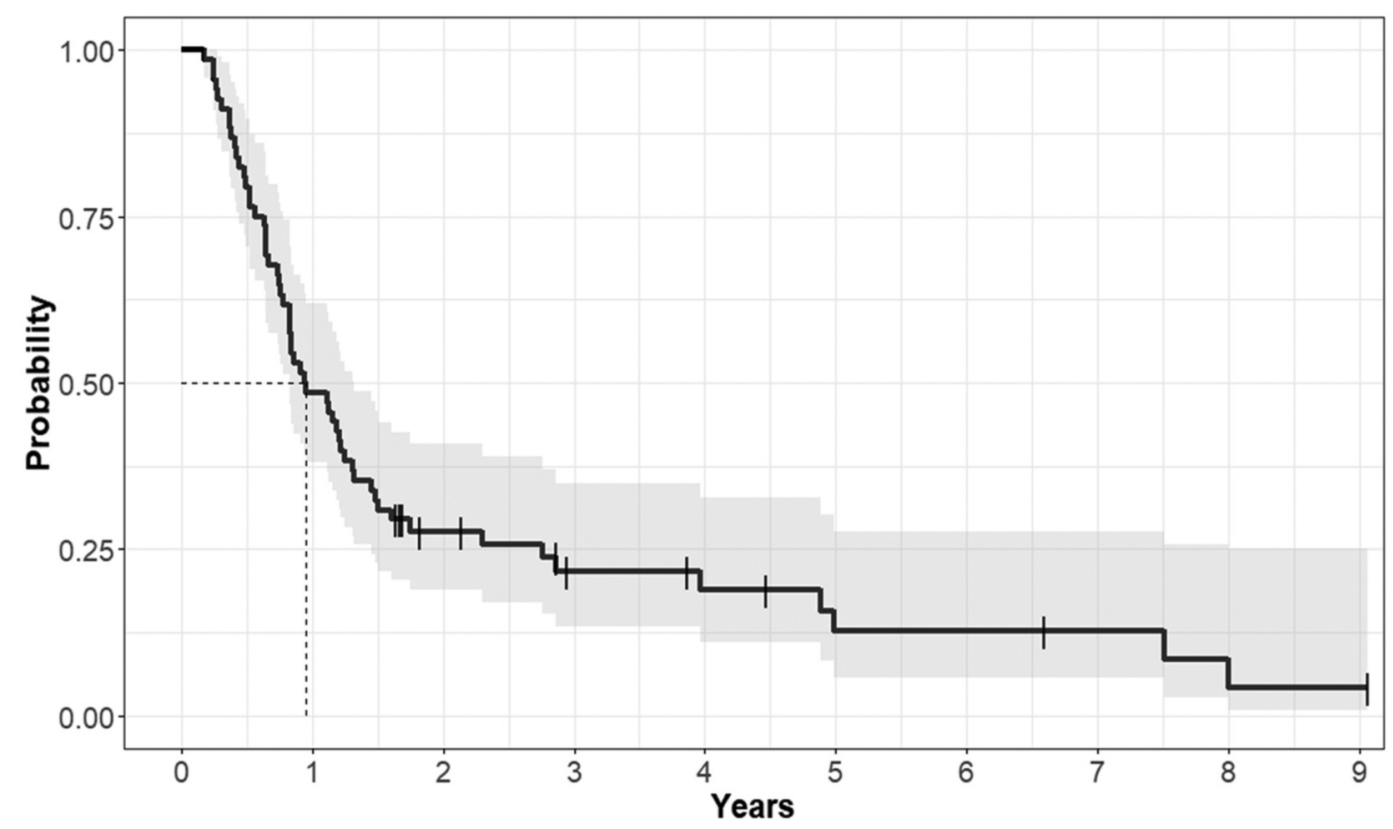

Figure 2. Overall survival curve of the entire patient population.

Table II. Multivariate analysis

\begin{tabular}{lccc}
\hline Variables & Hazard ratio & $\begin{array}{c}(95 \% \text { Confidence } \\
\text { interval })\end{array}$ & $p$-Value \\
\hline Age <65 years & 1.946 & $(1.069-3.542)$ & 0.029 \\
T3-4 & - & - & NS \\
N2-3 & 2.421 & $(1.374-4.265)$ & 0.002 \\
High LMRpre & 0.305 & $(0.090-1.037)$ & 0.057 \\
High NLRpre & 1.632 & $(0.903-2.948)$ & 0.105 \\
High LMRpost & - & - & NS \\
High NLRpost & 1.818 & $(1.043-3.168)$ & 0.035 \\
High PLRpost & - & - & NS \\
\hline
\end{tabular}

LMR: Lymphocyte-to-monocyte ratio; NLR: neutrophil-to-lymphocyte ratio; PLR: platelet-to-lymphocyte ratio.

at 1 year and 3 years were $48.5 \%$ and $21.6 \%$, respectively (Figure 2). In the univariate analysis, older age, higher $\mathrm{T}$ stage, higher N stage, higher NLRpre, lower LMRpre, higher PLRpost, higher NLRpost, and lower LMRpost were associated with poorer OS. Among the treatment factors, adjuvant chemotherapy showed improved OS in the univariate analysis, but a higher RT dose did not (Table I). We performed multivariate analyses for all the factors, except for the treatment factors. Finally, age, N stage, and NLRpost were confirmed as prognostic factors for OS (Table II, Figure 3A).

We classified patients into four groups according to the NLRpost and adjuvant chemotherapy, and then, confirmed significant differences (Figure 3B). Patients with lower NLRpost had better OS when adjuvant chemotherapy was administered. The median OS was 16.6 versus 4.8 months $(p<0.001)$ for lower NLRpost with adjuvant chemotherapy and lower NLRpost without adjuvant chemotherapy, respectively. In patients with higher NLRpost, the median OS was 12.4 with adjuvant chemotherapy and 4.5 months without adjuvant chemotherapy $(p=0.038)$. The improvement in OS was remarkable in patients with lower NLRpost and adjuvant chemotherapy.

Adverse effect. Lymphopenia was the most common and most severe hematologic adverse effect recorded after dCCRT wherein it was grade 2 in 19 patients $(28 \%)$, grade 3 in $18(26 \%)$, and grade 4 in $8(12 \%)$. Grade 2 anemia occurred in 19 patients $(28 \%)$ and grade 3 in $1(1 \%)$. Regarding neutropenia, 3 patients (4\%) experienced Grade 2, $2(3 \%)$ experienced Grade 3, and $3(4 \%)$ experienced Grade 4 . Lastly, grade 2 and 3 thrombocytopenia were both recorded in just 1 patient each $(1 \%)$.

\section{Discussion}

Inflammation and cancer are closely related; inflammatory TME promotes the proliferation and survival of malignant cells as well as angiogenesis and metastasis $(2,12)$. Testing leukocytes obtained from peripheral blood is a cost-effective and easy-to-use method to examine the systemic status of immune and inflammatory responses. 

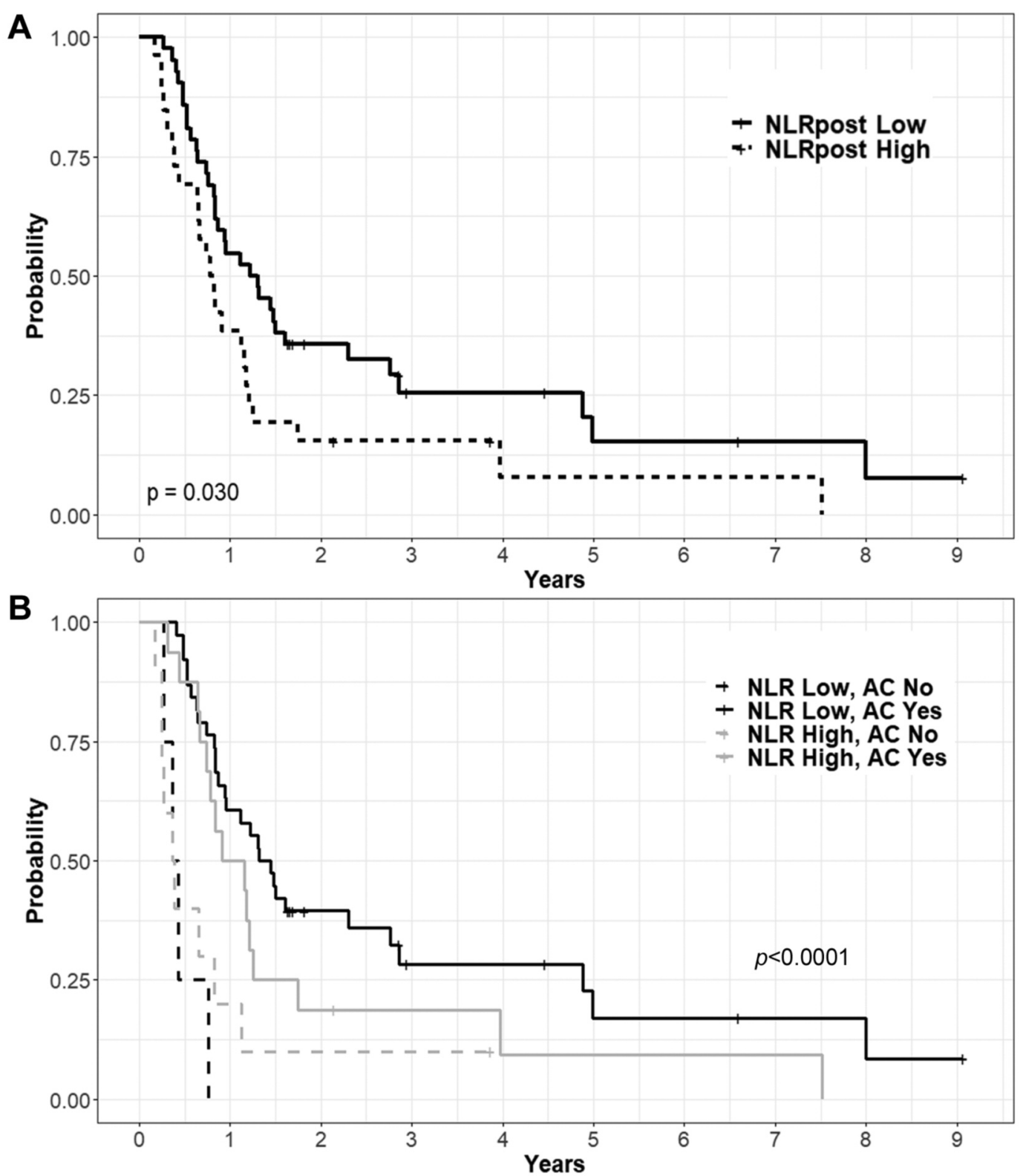

Figure 3. Overall survival curves according to the level of neutrophil-to-lymphocyte ratio (A) and adjuvant chemotherapy (B) after definitive concurrent chemoradiotherapy.

Among the peripheral blood cells, the main component of the anti-cancer immune response is the lymphocyte. Cytotoxic lymphocytes directly eradicate cancer cells via the death ligand/death receptor system and granular exocytosis (13). The presence of tumor-infiltrating lymphocytes, which can hinder tumor progression, is correlated with a good prognosis (14). In contrast, lymphopenia is associated with poor prognosis in solid and hematologic malignancies (15).

Neutrophils, monocytes, and platelets are also important elements of the anti-cancer immune response. In addition, neutrophils produce many kinds of pro-tumoral mediators, such as reactive oxygen species, elastase, integrins, angiogenic factors, and proteinases. Thus, neutrophils play an important role in genetic instability, tumor cell proliferation, angiogenesis, and metastasis (16, 17). Monocytes differentiate into macrophages and migrate into the tumor tissue. As tumor-associated macrophages, monocytes ultimately participate in tumor progression by immune regulation, angiogenesis, and tumor invasion (18). Platelets are correlated with tumor metastasis. Tumor cells can induce platelet aggregation by which platelet-associated angiogenesis-regulating factors are secreted (19). 
The absolute count of immune-inflammatory cells, which is affected by the myelosuppressive effect of chemotherapy (9), radiosensitivity of immune cells (8), and malnutrition due to the gastrointestinal adverse effects of dCCRT (20), should be cautiously interpreted. The absolute count of each cell shows a wide variation between patients, and changes with time even in a single patient. In contrast, relative stability could be expected when we use the ratio of immuneinflammatory cell counts, such as LMR, NLR, and PLR.

The prognostic value of the ratio of immune-inflammatory cell counts was examined in a pre-treatment setting rather than in a post-treatment setting. In this context, we compared LMR, NLR, and PLR in ESCC patients before and after dCCRT. We found that NLRpost predicts OS better than the others. As the absolute number of lymphocytes and neutrophils decreased after dCCRT, the higher NLRpost suggests that lymphocytes were affected relatively more than neutrophils. The anti-tumor activity of lymphocytes and the pro-tumor activity of neutrophils might accord with our results. To the best of our knowledge, this is the first study to evaluate the association of NLRpost and OS in ESCC patients who underwent dCCRT.

Inflammation induced by dCCRT has a yin-yang effect that can stimulate the rebound of residual malignant cells or improve therapeutic outcomes (12). NLRpost could be a good surrogate representing immune-inflammatory responses against malignant cells after the completion of dCCRT. The association between elevated NLRpost and poor survival has been reported in brain $(21)$, head and neck $(6,22)$, breast $(23)$, lung (24), and rectal (25) cancers. NLRpost has a potential benefit compared with NLRpre, which might be used to select patients who need additional therapy or intensive follow-up.

Sherry et al. (23) analyzed treatment outcomes of patients with stage II-III triple-negative breast cancer. NLR was calculated after the completion of RT following chemotherapy and breast surgery, and showed significant associations with locoregional failure and OS. Interestingly, among stage III breast cancer patients, patients with NLR $\geq 3$ experienced a higher rate of locoregional failure than those with NLR $<3$. Since hormone therapy is limited in triplenegative breast cancer patients, close monitoring of patients using post-treatment NLR might be helpful.

In ESCC patients, several studies reported survival benefit in the addition of chemotherapy to dCCRT $(10,26,27)$. However, adjuvant chemotherapy following dCCRT would not be administered to all ESCC patients considering the incidence of severe adverse effects $(28,29)$. Our study might contribute to the evaluation of the additional treatment group, since the benefit from adjuvant chemotherapy was notable in patients with NLRpost $\leq 5.44$ (Figure 2).

A limitation of the present study is the absence of serial laboratory data during the course of dCCRT. This potentially resulted from the retrospective study design of various institutions. The correlation between NLR dynamics and prognosis could be an independent theme for prospective studies. In addition, the functional classification of immune and inflammatory cells is becoming more sophisticated with the development of molecular biology. A future direction should be a collaborative system using NLR and molecular subtypes. Lastly, unsettled consensus of cutoff values of NLRpost is an important hurdle for clinical application.

\section{Conclusion}

We confirmed that NLRpost could be used for the prediction of prognosis in patients with ESCC who underwent dCCRT. A lower NLRpost score $(\leq 5.44)$ was associated with better OS. In addition, NLRpost might play a role in establishing an adjuvant therapy plan following dCCRT, given that the improvement in OS was remarkable in patients with lower NLRpost and who underwent adjuvant chemotherapy.

\section{Conflicts of Interest}

The Authors have no conflicts of interest to declare regarding this study.

\section{Authors' Contributions}

TK designed this study. HKK, YP, and TK collected and analyzed data. HJP, MYL, ARC, SH, and HB participated in the interpretation of results. HKK and YP drafted the article, and TK revised the article. All Authors read and approved the article.

\section{References}

1 Coussens LM, Zitvogel L and Palucka AK: Neutralizing tumorpromoting chronic inflammation: A magic bullet? Science 339(6117): 286-291, 2013. PMID: 23329041. DOI: 10.1126/ science. 1232227

2 Mantovani A, Allavena P, Sica A and Balkwill F: Cancer-related inflammation. Nature 454(7203): 436-444, 2008. PMID: 18650914. DOI: 10.1038 /nature07205

3 Pitt JM, Marabelle A, Eggermont A, Soria JC, Kroemer G and Zitvogel L: Targeting the tumor microenvironment: Removing obstruction to anticancer immune responses and immunotherapy. Ann Oncol 27(8): 1482-1492, 2016. PMID: 27069014. DOI: 10.1093/annonc/mdw168

4 Walsh SR, Cook EJ, Goulder F, Justin TA and Keeling NJ: Neutrophil-lymphocyte ratio as a prognostic factor in colorectal cancer. J Surg Oncol 91(3): 181-184, 2005. PMID: 16118772. DOI: $10.1002 /$ jso. 20329

5 Hu P, Shen H, Wang G, Zhang P, Liu Q and Du J: Prognostic significance of systemic inflammation-based lymphocytemonocyte ratio in patients with lung cancer: Based on a large cohort study. PLoS One 9(9): e108062, 2014. PMID: 25275631. DOI: 10.1371/journal.pone.0108062

6 Cannon NA, Meyer J, Iyengar P, Ahn C, Westover KD, Choy H and Timmerman R: Neutrophil-lymphocyte and plateletlymphocyte ratios as prognostic factors after stereotactic radiation therapy for early-stage non-small-cell lung cancer. $\mathrm{J}$ 
Thorac Oncol 10(2): 280-285, 2015. PMID: 25299234. DOI: 10.1097/JTO.0000000000000399

7 Yodying H, Matsuda A, Miyashita M, Matsumoto S, Sakurazawa N, Yamada $M$ and Uchida E: Prognostic significance of neutrophil-to-lymphocyte ratio and platelet-to-lymphocyte ratio in oncologic outcomes of esophageal cancer: A systematic review and meta-analysis. Ann Surg Oncol 23(2): 646-654, 2016. PMID: 26416715. DOI: 10.1245/s10434-015-4869-5

8 Zachariah B, Jacob SS, Gwede C, Cantor A, Patil J, Casey L and Zachariah AB: Effect of fractionated regional external beam radiotherapy on peripheral blood cell count. Int J Radiat Oncol Biol Phys 50(2): 465-472, 2001. PMID: 11380235. DOI: $10.1016 / \mathrm{s} 0360-3016(00) 01587-\mathrm{x}$

9 Crawford J, Dale DC and Lyman GH: Chemotherapy-induced neutropenia: Risks, consequences, and new directions for its management. Cancer 100(2): 228-237, 2004. PMID: 14716755. DOI: $10.1002 /$ cncr.11882

10 Koh HK, Park Y, Koo T, Park HJ, Lee MY, Chang AR, Hong S and Bae $\mathrm{H}$ : Adjuvant chemotherapy and dose escalation in definitive concurrent chemoradiotherapy for esophageal squamous cell carcinoma. Anticancer Res 40(3): 1771-1778, 2020. PMID: 32132086. DOI: 10.21873/anticanres.14131

11 R Development Core Team: R: A language and environment for statistical computing. Vienna, 2019. Available at: https://www.rproject.org/ [Last accessed on August 20, 2020]

12 Grivennikov SI, Greten FR and Karin M: Immunity, inflammation, and cancer. Cell 140(6): 883-899, 2010. PMID: 20303878. DOI: 10.1016/j.cell.2010.01.025

13 Martinez-Lostao L, Anel A and Pardo J: How do cytotoxic lymphocytes kill cancer cells? Clin Cancer Res 21(22): 5047-5056, 2015. PMID: 26567364. DOI: 10.1158/1078-0432.CCR-15-0685

14 Gooden MJ, de Bock GH, Leffers N, Daemen T and Nijman HW: The prognostic influence of tumour-infiltrating lymphocytes in cancer: A systematic review with meta-analysis. Br J Cancer 105(1): 93-103, 2011. PMID: 21629244. DOI: $10.1038 / \mathrm{bjc} .2011 .189$

15 Ray-Coquard I, Cropet C, Van Glabbeke M, Sebban C, Le Cesne A, Judson I, Tredan O, Verweij J, Biron P, Labidi I, Guastalla JP, Bachelot T, Perol D, Chabaud S, Hogendoorn PC, Cassier P, Dufresne A, Blay JY, European Organization for Research, Treatment of Cancer Soft Tissue and Bone Sarcoma Group: Lymphopenia as a prognostic factor for overall survival in advanced carcinomas, sarcomas, and lymphomas. Cancer Res 69(13): 5383-5391, 2009. PMID: 19549917. DOI: 10.1158/ 0008-5472.CAN-08-3845

16 Gregory AD and Houghton AM: Tumor-associated neutrophils: New targets for cancer therapy. Cancer Res 71(7): 2411-2416, 2011. PMID: 21427354. DOI: 10.1158/0008-5472.CAN-10-2583

17 Galdiero MR, Bonavita E, Barajon I, Garlanda C, Mantovani A and Jaillon S: Tumor associated macrophages and neutrophils in cancer. Immunobiology 218(11): 1402-1410, 2013. PMID: 23891329. DOI: 10.1016/j.imbio.2013.06.003

18 Lee HW, Choi HJ, Ha SJ, Lee KT and Kwon YG: Recruitment of monocytes/macrophages in different tumor microenvironments. Biochim Biophys Acta 1835(2): 170-179, 2013. PMID: 23287570. DOI: 10.1016/j.bbcan.2012.12.007

19 Yan $\mathrm{M}$ and Jurasz P: The role of platelets in the tumor microenvironment: From solid tumors to leukemia. Biochim Biophys Acta 1863(3): 392-400, 2016. PMID: 26193075. DOI: 10.1016/j.bbamcr.2015.07.008
20 Van Cutsem E and Arends J: The causes and consequences of cancer-associated malnutrition. Eur J Oncol Nurs 9: S51-63, 2005. PMID: 16437758. DOI: 10.1016/j.ejon.2005.09.007

21 Mason M, Maurice C, McNamara MG, Tieu MT, Lwin Z, Millar BA, Menard C, Laperriere N, Milosevic M, Atenafu EG, Mason W and Chung C: Neutrophil-lymphocyte ratio dynamics during concurrent chemo-radiotherapy for glioblastoma is an independent predictor for overall survival. J Neurooncol 132(3): 463-471, 2017. PMID: 28332000. DOI: 10.1007/s11060-017-2395-y

22 Ou D, Wang X, Wu M, Xue F, Li Y, Hu C and He X: Prognostic value of post-radiotherapy neutrophil-to-lymphocyte ratio in locally advanced nasopharyngeal carcinoma. Strahlenther Onkol 196(3): 252-261, 2020. PMID: 31701168. DOI: 10.1007/s00066019-01529-3

23 Sherry AD, von Eyben R, Newman NB, Gutkin P, Mayer I, Horst K, Chakravarthy AB and Rafat M: Systemic inflammation after radiation predicts locoregional recurrence, progression, and mortality in stage ii-iii triple-negative breast cancer. Int J Radiat Oncol Biol Phys 108(1): 268-276, 2020. PMID: 31809877. DOI: 10.1016/j.ijrobp.2019.11.398

24 Wang D, Guo D, Li A, Wang P, Teng F and Yu J: The posttreatment neutrophil-to-lymphocyte ratio and changes in this ratio predict survival after treatment of stage iii non-small-cell lung cancer with conventionally fractionated radiotherapy. Future Oncol 16(9): 439-449, 2020. PMID: 32141321. DOI: 10.2217/fon-2019-0837

25 Sung S, Son SH, Park EY and Kay CS: Prognosis of locally advanced rectal cancer can be predicted more accurately using pre- and post-chemoradiotherapy neutrophil-lymphocyte ratios in patients who received preoperative chemoradiotherapy. PLoS One 12(3): e0173955, 2017. PMID: 28291841. DOI: 10.1371/ journal.pone. 0173955

26 Fan CY, Su YF, Huang WY, Chao HL, Lin KT and Lin CS: Definitive radiotherapy dose escalation with chemotherapy for treating non-metastatic oesophageal cancer. Sci Rep 8(1): 12877, 2018. PMID: 30150679. DOI: 10.1038/s41598-018-31302-y

27 Wu SX, Li XY, Xu HY, Xu QN, Luo HS, Du ZS, Huang HC and $\mathrm{Wu} \mathrm{ZY}$ : Effect of consolidation chemotherapy following definitive chemoradiotherapy in patients with esophageal squamous cell cancer. Sci Rep 7(1): 16870, 2017. PMID: 29203855. DOI: 10.1038/s41598-017-17254-9

28 Kato K, Muro K, Minashi K, Ohtsu A, Ishikura S, Boku N, Takiuchi H, Komatsu Y, Miyata Y, Fukuda $\mathrm{H}$ and Gastrointestinal Oncology Study Group of the Japan Clinical Oncology G: Phase ii study of chemoradiotherapy with 5fluorouracil and cisplatin for stage ii-iii esophageal squamous cell carcinoma: Jcog trial (jcog 9906). Int J Radiat Oncol Biol Phys 81(3): 684-690, 2011. PMID: 20932658. DOI: 10.1016/ j.ijrobp.2010.06.033

29 Minsky BD, Pajak TF, Ginsberg RJ, Pisansky TM, Martenson J, Komaki R, Okawara G, Rosenthal SA and Kelsen DP: Int 0123 (radiation therapy oncology group 94-05) phase iii trial of combined-modality therapy for esophageal cancer: High-dose versus standard-dose radiation therapy. J Clin Oncol 20(5): 1167-1174, 2002. PMID: 11870157. DOI: 10.1200/JCO. 2002.20.5.1167

Received November 19, 2020

Revised November 30, 2020

Accepted December 1, 2020 